D. I. Cartwright and P. M. Soardi

Nagoya Math. J.

Vol. 102 (1986), 163-180

\title{
RANDOM WALKS ON FREE PRODUCTS, QUOTIENTS AND AMALGAMS
}

\author{
DONALD I. CARTWRIGHT AND P. M. SOARDI
}

\section{\$1. Introduction}

Suppose that $G$ is a discrete group and $p$ is a probability measure on $G$. Consider the associated random walk $\left\{X_{n}\right\}$ on $G$. That is, let $X_{n}=Y_{1} Y_{2} \cdots Y_{n}$, where the $Y_{j}^{\prime}$ 's are independent and identically distributed $G$-valued variables with density $p$. An important problem in the study of this random walk is the evaluation of the resolvent (or Green's function) $R(z, x)$ of $p$. For example, the resolvent provides, in principle, the values of the $n$ step transition probabilities of the process, and in several cases knowledge of $R(z, x)$ permits a description of the asymptotic behaviour of these probabilities.

When $G$ is abelian, the methods of Fourier analysis are available, but, generally, new methods must be found to study the non-commutative case (see [15], however, where explicit knowledge of the representations of a free group allows an extension of the Fourier methods). When $G$ is thought of in terms of generators and relations, a natural point of departure is the study of free groups or, more generally, groups which are free products of "smaller" groups. On such groups, the most natural probabilities $p$ are those which generate "nearest neighbour" random walks.

Random walks on finitely generated free groups have been studied from many points of view. One of the earliest papers on this subject is [13]. Using the idea of the length $|x|$ of an element $x$, nearest neighbour random walks (for which $p(x)=0$ if $|x|>1$ ) have been considered in [12] and [10], for example. Isotropic random walks (in which $p(x)$ depends only on $|x|)$ were studied in [15], [17] and [9]. See also [1] and [4].

For finite free products of finite groups, random walks and related subjects have been studied in, e.g., [2], [5] and [22]. In a recent paper

Received March 4, 1985. 
[20], the free product of two arbitrary discrete groups is considered, and a local limit theorem is obtained for the analogue of a nearest neighbour random walk.

A natural next step is to free products with amalgamation. One paper in this direction is [16].

Let us briefly describe the contents of the present paper. After establishing our notation in Section 2, we determine in Section 3 the resolvent for "nearest neighbour" random walks on the free product of a finite or countable family of discrete groups. It turns out to be a sort of "free product" of the resolvents on the component groups. In Section 4 , this is applied to obtain a local limit theorem for the case when each factor group $G_{j}$ is finite and $p$ is constant on each $G_{j} \backslash\{e\}$. We also obtain a quick derivation of the "Plancherel measure" of $p$ in two cases which had been studied in several earlier papers. In Section 5 , we consider a discrete group $G$ having a finite normal subgroup $H$, and show how to find the resolvent of a probability obtained from a probability on $G / H$ and one of $H$. We apply this to the case when $G$ is the free product with amalgamation $*_{H} G_{j}$ of a family of discrete groups $G_{j}$ having a common finite normal subgroup $H$. As well as proving an appropriate local limit theorem, we are able to determine the orthogonal polynomials of the Plancherel measure of a symmetric probability on $G$ obtained from ones on $G / H$ and $H$.

\section{$\S 2$. Notation and Definitions}

Let $G$ be a discrete group and $p$ a probability measure on $G$, i.e. a function $p: G \rightarrow R$ such that $p(x) \geqq 0$ for all $x \in G$ and such that $\sum_{x \in G} p(x)$ $=1$. Associated to $p$ there is a random walk $\left\{X_{n}: n=0,1, \cdots\right\}$ on $G$ for which $P\left(X_{n+1}=y \mid X_{n}=x\right)=p\left(x^{-1} y\right)$ for all $x, y \in G$ and $n \geqq 0$. Alternatively, we can associate a directed graph $\Gamma$ to $(G, p)$ in the following way. The set $V$ of vertices of $\Gamma$ is $G$, and there is an edge from $x$ to $y$ (one only) if $p\left(x^{-1} y\right)>0$. We can then interpret the above process $\left\{X_{n}\right\}$ as a random walk on $V$ in which at each step one goes from a vertex $x$ to one of the neighbouring vertices $y$ with probability $p\left(x^{-1} y\right)$.

Let $T$ denote the convolution operator $f \mapsto p * f$ on $\ell^{2}(G)$. We denote the spectrum of $T$ by $X$. If $z \in C \backslash X$, then $(z I-T)^{-1}$ is also a convolution operator $f \mapsto R(z) * f$, and the function $R(z)$ is called the relsolvent or the Green function of $p$. It satisfies $R(z) \in \ell^{2}(G)$ and $R(z) *\left(z \delta_{e}-p\right)=\delta_{e}$, 
where $\delta_{e}(x)=1$ if $x=e$ and $\delta_{e}(x)=0$ otheriswe. Writing $R(z, x)$ for the value of $R(z)$ at $x \in G$ and $r$ for $\max \{|t|: t \in X\}$, we have

$$
R(z, x)=\sum_{n=0}^{\infty} \frac{p^{* n}(x)}{z^{n+1}} \quad \text { if } x \in G \text { and }|z|>r
$$

where $p^{* n}$ is the $n$th convolution power of $p\left(p^{* 0}=\delta_{e}\right)$. Note that $p^{* n}(x)$ $=P\left\{X_{n}=x \mid X_{0}=e\right\}$, the probability of being at $x$ after $n$ steps, starting at $e$. The value $R(z, e)$ of $R(z)$ at $e$ is denoted by $S(z)$. The number $r$ is called the spectral radius of $p$.

When $p$ is symmetric, i.e. $p\left(x^{-1}\right)=p(x)$ for all $x \in G$, the operator $T$ is hermitian and $X \subseteq[-1,1]$. Each operator $S$ in the operator norm closed algebra $A^{*}$ generated by $T$ is a convolution operator $f \mapsto g * f$. Identifying $S$ and $g$, we can regard $A^{*}$ as a convolution algebra of functions in $\ell^{2}(G)$. In particular, $T$ is identified with $p$ and $I$ with $\delta_{e}$. Now $A^{*}$ is a $C^{*}$-algebra, and we may consider the Gelfand isomorphism $\wedge: A^{*} \rightarrow \mathscr{C}(X)$, where $\mathscr{C}(X)$ is the algebra of continuous functions $X \rightarrow C$. A probability measure $\mu$ is defined on $X$ by the formula

$$
f(e)=\int_{X} \hat{f} d \mu \quad\left(f \in A^{*}\right) .
$$

In particular, $R(z) \in A^{*}$, and so

$$
S(z)=R(z, e)=\int_{X} \frac{d \mu(t)}{z-t} \quad \text { if } z \in C \backslash X .
$$

Thus $S(z)$ is the Stieltjes transform of $\mu$. The measure $\mu$ is called the Plancherel measure of $p$.

Throughout this paper, when various probabilities such as $p_{j}, \tilde{p}_{j}$, etc., are discussed, the corresponding resolvents will be denoted by $R_{j}(z), \tilde{R}_{j}(z)$, etc., usually without explanation. The same applies to the corresponding $S(z), X$ and $r$, as well as $\mu$ in the symmetric case.

\section{§ 3. The resolvent for free products of discrete groups}

Let $G$ be the free product of a finite or countable family $\left\{G_{j}\right\}_{j \in J}$ of discrete groups. Each $x \in G, x \neq e$, may be written in a unique way as a reduced word, i.e. a product $x_{i_{1}} x_{i_{2}} \cdots x_{i_{n}}$, where $e \neq x_{i_{\nu}} \in G_{i_{\nu}}$ for each $\nu$ and $i_{\nu} \neq i_{\nu+1}$ for $1 \leqq \nu<n$. For each $j \in J$, let $p_{j}$ be a probability measure on $G_{j}$. We extend $p_{j}$ to $G$ by defining $p_{j}(x)=0$ if $x \in G \backslash G_{j}$. Let $\alpha_{j}>0$ be numbers such that $\Sigma_{j} \alpha_{j}=1$, and define a probability measure $p$ on $G$ 
by $p(x)=\sum_{j} \alpha_{j} p_{j}(x)$. This measure corresponds to the random walk on $G$ in which the possible moves from a point $x \in G$ are to points $x x_{j}, x_{j} \in G_{j}$ for some $j$. This process can be thought of as a generalized simple random walk on $G$. Our first theorem shows that the resolvent of $p$ can be expressed in a remarkably simple way in terms of the resolvents of the probabilities $p_{j}$.

We first need two lemmas.

LEMMA 1. Let $R(z)$ be the resolvent of a probability measure $p$ on a discrete group $G$, and let $S(z)=R(z, e)$. Then there is a constant $M_{0}$, independent of $G$ and $p$, such that $|R(z, x)| \leqq|S(z)|$ whenever $x \in G$ and $|z| \geqq M_{0}$.

Proof. Let $|z|>1$. Then $\|R(z)\|_{2} \leqq \sum_{n=0}^{\infty}\left\|p^{* n}\right\|_{2} /|z|^{n+1} \leqq 1 /(|z|-1)$ by equation (2.1), since $\left\|p^{* n}\right\|_{2} \leqq\left\|p^{* n}\right\|_{1}=1$. Also,

$|S(z)| \geqq 1 /|z|-\sum_{n=1}^{\infty}\left|p^{* n}(e) / z^{n+1}\right| \geqq 1 /|z|-1 /|z|(|z|-1)=(|z|-2) /|z|(|z|-1)$.

Therefore $\|R(z)\|_{2}^{2} /|S(z)|^{2} \leqq|z|^{2} /(|z|-2)^{2} \leqq 2$ once $|z| \geqq 4+2 \sqrt{2},=M_{0}$ say. Thus $|R(z, x)|^{2} \leqq\|R(z)\|_{2}^{2}-|S(z)|^{2} \leqq|S(z)|^{2}$ for $x \neq e$ once $|z| \geqq M_{0}$.

LEMMA 2. Let $R(z)$ be the resolvent of a probability measure $p$ on $a$ discrete group $G$ and let $S(z)=R(z, e)$. Let $0<\alpha \leqq 1$, Then for $|w|>6$, the equation $\alpha / S(\lambda)=w$ has a unique solution $\lambda=\lambda(w)$ satisfying $|\lambda|>3$. Furthermore, $w \mapsto \lambda(w)$ is an analytic function on $\{w \in C:|w|>6\}$, and satisfies $|\lambda(w)| \geqq|w| / 2$ and

$$
|\alpha \lambda(w)-w| \leqq 3 \alpha
$$

Proof. Set $f(u)=S(1 / u)$ and $f(0)=0$. Then $f(u)=\sum_{n=0}^{\infty} p^{* n}(e) u^{n+1}$, and, as in Lemma $1,|f(u)| \geqq|u|-\sum_{n=1}^{\infty}\left|p^{* n}(e) u^{n+1}\right| \geqq|u|(1-2|u|) /(1-|u|)$. Thus $|f(u)| \geqq 1 / 6$ if $|u|=1 / 3$, and $f(u) \neq 0$ if $0<|u| \leqq 1 / 3$. Because also $f^{\prime}(0)=1$, the inverse function theorem shows that $f^{-1}(v)$ exists and is analytic for $v$ in a neighbourhood of 0 , and in fact, one can show, for $|v|<1 / 6$.

If $v=f(u)$ and $0<|u| \leqq 1 / 3$, then $|v / u-1| \leqq\left.\sum_{n=1}^{\infty}\left|p^{* n}(e) u^{n} \leqq \sum_{n=1}^{\infty}\right| u\right|^{n}$ $\leqq 3|u| \mid 2 \leqq 1 / 2$. Hence $|v / u| \geqq 1 / 2$ and $|v / u-1| \leqq 3|v|$, from which the result follows.

If $G$ is the free product of the groups $G_{j}$, we apply Lemma 2 to the resolvent $R_{j}(z)$ of each $p_{j}$ to obtain an analytic function $\lambda_{j}(w)$ for $6<|w|$ $<\infty$, such that 


$$
\alpha_{j} / S_{j}\left(\lambda_{j}(w)\right)=w .
$$

The estimate (3.1) shows that the series $\Sigma_{j}\left(\alpha_{j} \lambda_{j}(w)-w\right)$ represents an analytic function, bounded by $\sum_{j} 3 \alpha_{j}=3$, for $6<|w|<\infty$. The equation

$$
z=w+\Sigma_{j}\left(\alpha_{j} \lambda_{j}(w)-w\right)
$$

defines $w$ implicitly as a function $w(z)$ of $z$, for $M_{1}<|z|<\infty$, say, such that $w(z) \rightarrow \infty$ as $z \rightarrow \infty$. For if we write $z=1 / t$ and $w=1 / v$, equation (3.3) takes the form $t=h(v)$, where $h$ is analytic for $|v|<1 / 6$ with $h(0)=0$ and $h^{\prime}(0)=1$. Combining the above observations, we obtain analytic functions $\lambda_{j}$ of $z$ for $M_{1}<|z|<\infty$ such that $\left|\lambda_{j}(z)\right| \geqq|w(z)| / 2 \geqslant(|z|-3) / 2$. We can now state the main result of this section.

Theorem 1. Suppose that $G$ is the free product of a finite or countable family $\left\{G_{j}, j \in J\right\}$ of discrete groups. Form the probability $p=\Sigma_{j} \alpha_{j} p_{j}$ on $G$, where $\alpha_{j}>0$ and $\Sigma_{j} \alpha_{j}=1$, using the probabilities $p_{j}$ on $G_{j}$, as above, and let $\lambda_{j}$ and $w$ be the analytic functions of $z$ defined via (3.2) and (3.3). Then for some constant $M$ the resolvent of $p$ is given by

$$
R(z, x)=S(z) g(z, x) \quad \text { for } x \in G \text { and }|z| \geqq M,
$$

where $g(z, x)$ is defined as follows: $g(z, e)=1$, and if $x=x_{j_{1}} x_{j_{2}} \cdots x_{j_{m}}$ is any reduced word in $G$, then $g(z, x)=g_{j_{1}}\left(x_{j_{1}}\right) g_{j_{2}}\left(x_{j_{2}}\right) \cdots g_{j_{m}}\left(x_{j_{m}}\right)$, where $g_{j}\left(x_{j}\right)$ $=R_{j}\left(\lambda_{j}, x_{j}\right) / S_{j}\left(\lambda_{j}\right)$.

Furthermore, $S(z)=1 / w(z)$.

Proof. We choose $M \geqq M_{1}$ so large that $(M-3) / 2 \geqq M_{0}$, where $M_{0}$ is the constant in Lemma 1 , and $M_{1}$ was defined after (3.3). Fix $z$ with $|z| \geqq M$, and write $g(x)$ for $g(z, x)$. Now $\left|\lambda_{j}(z)\right| \geqq(|z|-3) / 2 \geqq M_{0}$, so that $\left|g_{j}\left(x_{j}\right)\right| \leqq 1$ for each $j$ and each $x_{j} \in G_{j}$, by Lemma 1 . Therefore $g$ is a bounded function on $G$, so that $p * g$ and $p_{j} * g$ are defined. If $x=x_{i_{1}} x_{i_{2}}$ $\cdots x_{i_{m}}$ is a reduced word, then $\left(p_{j} * g\right)(x)=\left(p_{j} * g_{j}\right)(e) g(x)=\left(\lambda_{j}-1 / S_{j}\left(\lambda_{j}\right)\right) g(x)$ if $j \neq i_{1}$, while $\left(p_{j} * g\right)(x)=\left(p_{i_{1}} * g_{i_{1}}\right)\left(x_{i_{1}}\right) g_{i_{2}}\left(x_{i_{2}}\right) \cdots g_{i_{n}}\left(x_{i_{n}}\right)=\lambda_{i_{1}} g_{i_{1}}\left(x_{i_{1}}\right) g_{i_{2}}\left(x_{i_{2}}\right)$ $\cdots g_{i_{n}}\left(x_{i_{n}}\right)=\lambda_{i_{1}} g(x)$ if $j=i_{1}$. Therefore

$$
\begin{aligned}
(p * g)(x)=\Sigma_{j} \alpha_{j}\left(p_{j} * g\right)(x) & =\left[\alpha_{i_{1}} \lambda_{i_{1}}+\sum_{j \neq i_{1}} \alpha_{j}\left(\lambda_{j}-1 / S_{j}\left(\lambda_{j}\right)\right)\right] g(x) \\
& =\left[\alpha_{i_{1}} / S_{i_{1}}\left(\lambda_{i_{1}}\right)+\Sigma_{j}\left(\alpha_{j} \lambda_{j}-\alpha_{j} / S_{j}\left(\lambda_{j}\right)\right)\right] g(x) \\
& =\left[w+\Sigma_{j}\left(\alpha_{j} \lambda_{j}-w\right)\right] g(x) \\
& =z g(x) .
\end{aligned}
$$

Thus $(p * g)(x)=z g(x)$ for each $x \neq e$, while 


$$
\begin{aligned}
(p * g)(e)=\Sigma_{j} \alpha_{j}\left(p_{j} * g_{j}\right)(e) & =\Sigma_{j} \alpha_{j}\left(\lambda_{j}-1 / S_{j}\left(\lambda_{j}\right)\right) \\
& =z-w .
\end{aligned}
$$

Thus $h(x)=g(x) / w$ is a bounded function satisfying $\left(z \delta_{e}-p\right) * h=\delta_{e}$. Now $|z|>1$, so that $\left(z \delta_{e}-p\right)^{-1}$ exists and is in $\ell^{1}(G)$. Therefore $\left(z \delta_{e}-p\right)^{-1}$ $=\left(z \delta_{e}-p\right)^{-1} * \delta_{e}=\left(z \delta_{e}-p\right)^{-1} *\left(\left(z \delta_{e}-p\right) * h\right)=\left(\left(z \delta_{e}-p\right)^{-1} *\left(z \delta_{e}-p\right)\right) * h$ $=\delta_{e} * h=h$. That is, $R(z, x)=g(x) / w$. In particular, $S(z)=1 / w$.

Remark. Nowhere in the above proof do we use the hypothesis that $p(x) \geqq 0$ for all $x \in G$. We need only assume that $\sum_{x \in G}|p(x)| \leqq 1$.

\section{$\S 4$. The free product of a family of finite groups; A local limit theorem}

§4.1. An important special case of Theorem 1 occurs when all the groups $G_{j}$ are finite, with $\left|G_{j}\right|=r_{j}(\geqq 2)$, say, and when $p_{j}(x)=1 /\left(r_{j}-1\right)$ for each $x \in G_{j}, x \neq e$. This situation has been studied (for a finite number of groups $G_{j}$ ) in [5], [10] and [13], for example. Using Theorem 1, we shall be able to prove the following local limit theorem. For convenience we shall write $|e|=0$ and $|x|=m$ if $x \in G$ is a reduced word $x_{i_{1}} x_{i_{2}} \cdots x_{i_{m}}$.

TheOREM 2. Let $G$ be the free product of a finite or countable family $\left\{G_{j}, j \in J\right\}$ of finite groups. Form the probability $p=\Sigma \alpha_{j} p_{j}$ on $G$ of the probability measures $p_{j}$ on $G_{j}$ described above. Let $X \subseteq[-1,1]$ be the spectrum associated with $p$, and let $b=\max X$ and $a=\min X$.

i) If $r_{j} \neq 2$ for some $j \in J$, then $0<-a<b<1$ and for each fixed $x \in G$, we have

$$
p^{* n}(x)=\phi(x) n^{-3 / 2} b^{n}\left(1+O\left(\frac{1}{n}\right)\right) \quad \text { as } n \rightarrow \infty
$$

for some $\phi(x)>0$.

ii) If $r_{j}=2$ for all $j \in J$, and if $|J|>2$, the $-a=b<1$ and for each fixed $x \in G$ we have (4.1) as $n \rightarrow \infty$ with $n \equiv|x| \bmod 2$, and $p^{* n}(x)=0$ if $n \not|x| \bmod 2$.

iii) If $r_{j}=2$ for each $j \in J$ and if $|J|=2$, then $-a=b=1$ and

$$
p^{* n}(x)=\frac{1}{\sqrt{ } 2 \pi \alpha_{1} \alpha_{2}} n^{-1 / 2}+O\left(n^{-3 / 2}\right)
$$

as $n \rightarrow \infty$ with $n \equiv|x| \bmod 2$, and $p^{* n}(x)=0$ if $n \not|x|(\bmod 2)$.

We have included case (iii) of Theorem 2 for completeness although 
it appears more or less explicitly in [11] and [15].

Let us first calculate the resolvent for each group $G_{j}$.

Lemma 3. Let $G$ be a finite group, with $|G|=r$, and let $p(x)=1 /(r-1)$ for each $x \in G, x \neq e$. Then $R(z, x)=1 /(z-1)((r-1) z+1)$ if $x \neq e$, while $S(z)=((r-1)(z-1)+1) /(z-1)((r-1) z+1)$.

Proof. Now $(p * f)(x)=\sum_{e \neq y \in G} f(y x)(r-1)=\left(\sum_{u \in G} f(u)\right) /(r-1)-$ $f(x) /(r-1)=\sigma-f(x) /(r-1)$, say. Thus $\left(z \delta_{e}-p\right) * f=\delta_{e}$ means that $(z+1 /(r-1)) f(x)=\sigma$ if $x \neq e$, and $(z+1 /(r-1)) f(e)=\sigma+1$. Therefore $(r-1) \sigma=\sum_{x \in G} f(x)=((r-1) \sigma+\sigma+1) /(z+1 /(r-1))$, so that $\sigma=$ $1 /(r-1)(z-1)$. The result now follows.

The equations $\alpha_{j} / S_{j}\left(\lambda_{j}\right)=w$ become in this case

$$
\alpha_{j}\left(r_{j}-1\right) \lambda_{j}^{2}-\left(\alpha_{j}\left(r_{j}-2\right)+w\left(r_{j}-1\right)\right) \lambda_{j}+w\left(r_{j}-2\right)-\alpha_{j}=0,
$$

which have solutions

$$
\lambda_{j}(w)=\frac{\alpha_{j}\left(r_{j}-2\right)+w\left(r_{j}-1\right)+\sqrt{\left.w\left(r_{j}-1\right)-\alpha_{j}\left(r_{j}-2\right)\right)^{2}+4 \alpha_{j}^{2}\left(r_{j}-1\right)}}{2 \alpha_{j}\left(r_{j}-1\right)},
$$

where the square root is chosen so as to behave like $w\left(r_{j}-1\right)$ as $w \rightarrow \infty$. Thus $\lambda_{j}(w)$ is the solution described in Lemma 2. We can define the functions $\lambda_{j}(w)$ simultaneously on $C \backslash \Gamma$, where $\Gamma$ is a simple path passing through all the points $\alpha_{j}\left(r_{j}-2\right) /\left(r_{j}-1\right) \pm 2 i \alpha_{j} / \sqrt{r_{j}-1}$ and meeting the $x$-axis only at the origin. Note that the square root in (4.2) is positive for $w \in(0, \infty)$ and negative for $w \in(-\infty, 0)$.

Equation (3.3) in this case takes the form, writing $\beta_{j}$ for $\alpha_{j} /\left(r_{j}-1\right)$.

$$
z=w+(1 / 2) \Sigma_{j}\left\{\beta_{j}\left(r_{j}-2\right)-w+\sqrt{\left(w-\beta_{j}\left(r_{j}-2\right)\right)^{2}+4 \beta_{j}^{2}\left(r_{j}-1\right)}\right\}
$$

As explained before Theorem 1, this series converges for $|w|$ large. In fact, it converges for $w$ in a neighbourhood $O_{+}$of $(0, \infty)$ in $C$. Indeed, $\operatorname{Re} \sqrt{\left(w-\beta_{j}(r-2)\right)^{2}+4 \beta_{j}^{2}\left(r_{j}-1\right)}=0$ if and only if

$$
w \in L_{j}=\left\{\beta_{j}\left(r_{j}-2\right)+i y:|y| \geqq 2 \beta_{j} \sqrt{r_{j}-1}\right\} .
$$

So all the square roots in (4.3) have real part $>0$ if $\operatorname{Re} w>0$ and $w \notin$ $\cup_{j} L_{j}$. Let $O_{+}=\left\{w \in C \backslash \Gamma: \operatorname{Re}(w)>0\right.$ and $\left.w \notin \cup_{j} L_{j}\right\}$. If $w \in O_{+}$, the $j$ th summand in (4.3) equals

$$
4 \beta_{j}^{2}\left(r_{j}-1\right) /\left(w-\beta_{j}\left(r_{j}-2\right)+\sqrt{\left(\beta_{j}\left(r_{j}-2\right)-w\right)^{2}+4 \beta_{j}^{2}\left(r_{j}-1\right)}\right),
$$

and here the denominator has real part at least $\operatorname{Re}(w)-\beta_{j}(r-2)$. Thus 
the series in (4.3) converges if $w \in O_{+}$. Similarly the series converges if $w \in O_{-}=\left\{w \in C \backslash \Gamma: \operatorname{Re} w<0\right.$ and $\left.-w \notin \cup_{j} L_{j}\right\}$, a neighbourhood of $(-\infty, 0)$. Let $f(w)$ denote the function defined by the right hand side of (4.3) for $w \in O_{+} \cup O_{-}$.

If $w \in(0, \infty)$, one can easily check that $f(w)>0$ and $f^{\prime \prime}(w)>0$, and that $f^{\prime}(w) \rightarrow 1$ as $w \rightarrow \infty$ and $f^{\prime}(w) \rightarrow 1-\Sigma_{j}\left(r_{j}-1\right) / r_{j}$ as $w \rightarrow 0$. Unless $G$ is the free product of two groups of order 2 , we have $1-\Sigma_{j}\left(r_{j}-1\right) / r_{j}<0$, and so $f$ has a unique minimum point $w_{+} \in(0, \infty)$. Similarly, for $w \in$ $(-\infty, 0)$ we have $f(w)<0$ and $f^{\prime \prime}(w)<0$, and $f^{\prime}(w) \rightarrow 1$ as $w \rightarrow-\infty$ and $f^{\prime}(w) \rightarrow 1-\Sigma_{j} 1 / r_{j}$ as $w \rightarrow 0$. So if $\Sigma 1 / r_{j}>1$ then $f$ has a unique maximum point $w_{-} \in(-\infty, 0)$, while if $\Sigma_{j} 1 / r_{j} \leqq 1$ then $f$ is strictly increasing on $(-\infty, 0)$, in which case we write $w_{-}=0$ and $f\left(w_{-}\right)=-\Sigma_{j} \beta_{j}$.

Lemma 4. We have $a=f\left(w_{-}\right)$and $b=f\left(w_{+}\right)$unless $G$ is the free product of two groups of order 2 , in which case $b=-a=1$.

Proof. We first show that $S$ is not regular at $a$ and $b$. Suppose, by way of contradiction, that $S$ could be defined analytically in a neighbourhood $|z-b|<\varepsilon$ of $b$. Since $\overline{S(\bar{z})}=S(z)$ for $z \in C \backslash X$, we must have $S(x) \in \boldsymbol{R}$ for $x \in(b-\varepsilon, b+\varepsilon)$. The Stieltjes Inversion Formula [6, p. 90] tells us that $\mu((b-\varepsilon, b+\varepsilon))=0$, which contradicts the fact that $b \in X$ $=\operatorname{Supp}(\mu)$. The same argument shows that $a$ is singular for $S$.

Now Theorem 1 tells us that $z=f(1 / S(z))$ for $z$ large, and so, by analytic continuation, also for $z \in(b, \infty)$. Furthermore, $z=f(1 / S(z))$ is satisfied for $z>z_{+}=f\left(w_{+}\right)$because $f^{\prime}(w) \neq 0$ for $w>w_{+}$. Moreover, $S(z)$ is not regular at $z_{+}$, for otherwise we would obtain the equation $f(1 / S(z))=z$ for $z$ near $z_{+}$, which is impossible because $f^{\prime}\left(w_{+}\right)=0$. We then conclude that $b=z_{+}$. The same argument shows that $a=f\left(w_{-}\right)$, with an obvious modification in the case $w_{-}=0$. Similarly, slight modifications to the above argument show that $b=-a=1$ when $G$ is the free product of two groups of order 2 .

Lemma 5. For $w \in(0, \infty)$ we have $f(w) \geqq-f(-w)$, and thus $b \geqq-a$. Strict inequality holds unless $r_{j}=2$ for all $j$.

Proof. If $w \in(0, \infty)$, we have

$$
f(w)=w+(1 / 2) \Sigma_{j}\left\{\beta_{j}\left(r_{j}-2\right)-w+\sqrt{\left(w-\beta_{j}\left(r_{j}-2\right)\right)^{2}+4 \beta_{j}^{2}\left(r_{j}-1\right)}\right\}
$$

and 


$$
f(-w)=-w+(1 / 2) \Sigma_{j}\left\{\beta_{j}\left(r_{j}-2\right)+w-\sqrt{\left(w+\beta_{j}\left(r_{j}-2\right)\right)^{2}+4 \beta_{j}^{2}\left(r_{j}-1\right)},\right.
$$

where the square roots are now all positive. But

$$
\begin{aligned}
& \sqrt{\left(\beta_{j}\left(r_{j}-2\right)+w\right)^{2}+4 \beta_{j}^{2}\left(r_{j}-1\right)} \\
& \quad \leqq \sqrt{\left(\beta_{j}\left(r_{j}-2\right)-w\right)^{2}+4 \beta_{j}^{2}\left(r_{j}-1\right)}+2 \beta_{j}\left(r_{j}-2\right),
\end{aligned}
$$

and so $-f(-w) \leqq f(w)$. By Lemma 4 , we have $b=f\left(w_{+}\right) \geqq-f\left(-w_{+}\right) \geqq$ $-f\left(w_{-}\right)=-a$. The inequality (4.4) is strict if $r_{j} \neq 2$, and so $f(w)>-f(w)$ for $w \in(0, \infty)$, and $b>-a$ unless all $r_{j}$ 's equal 2 .

Proof of Theorem 2. We apply Darboux's method to

$$
\sum_{n=0}^{\infty} p^{* n}(x) u^{n+1}=R\left(\frac{1}{u}, x\right) .
$$

If $x=x_{i_{1}} \cdots x_{i_{m}}$ is a reduced word in $G$, then by Theorem 1 ,

$$
R\left(\frac{1}{u}, x\right)=\frac{1}{w} r_{i_{1}}(w) r_{i_{2}}(w) \cdots r_{i_{m}}(w),
$$

where $r_{i_{\nu}}(w)=R_{i_{\nu}}\left(\lambda_{i_{\nu}}(w), x_{i_{\nu}}\right) / S_{i_{\nu}}\left(\lambda_{i_{\nu}}(w)\right)$ and where $w$ satisfies $1 / u=f(w)$. By Lemma 3 and formula (4.2),

$$
\begin{aligned}
r_{j}(w) & =1 /\left(\left(r_{j}-1\right)\left(\lambda_{j}(w)-1\right)+1\right) \\
& =\left\{\beta_{j}\left(r_{j}-2\right)-w+\sqrt{\left(w-\beta_{j}\left(r_{j}-2\right)\right)^{2}+4 \beta_{j}^{2}\left(r_{j}-1\right)}\right\} / 2 \beta_{j}\left(r_{j}-1\right),
\end{aligned}
$$

which has an expansion

$$
r_{j}(w)=r_{j}\left(w_{+}\right)+r_{j}^{\prime}\left(w_{+}\right)\left(w-w_{+}\right)+\cdots
$$

about $w_{+}$unless we are in case (iii) of the theorem. Notice that $r_{j}\left(w_{+}\right)$ $>0$ and $r_{j}^{\prime}\left(w_{+}\right)<0$. Thus $r_{i_{1}}(w) r_{i_{2}}(w) \cdots r_{i_{m}}(w)=A_{0}+A_{1}\left(w-w_{+}\right)+\cdots$, where

$$
A_{0}=\prod_{\nu=1}^{m} r_{i_{\nu}}\left(w_{+}\right)>0 \text { and } A_{1}=A_{0} \sum_{\nu=1}^{m} r_{i_{\nu}}^{\prime}\left(w_{+}\right) / r_{i_{\nu}}\left(w_{+}\right)<0 .
$$

Now $1 / u=f(w)=f\left(w_{+}\right)+(1 / 2) f^{\prime \prime}\left(w_{+}\right)\left(w-w_{+}\right)^{2}+\cdots$ and $f\left(w_{+}\right)=b$. So $w$ may be expanded in a power series in $((1-u b) / u)^{1 / 2}$, and thus in $\sqrt{1-u b}$ :

$$
w=w_{+}+\sqrt{\frac{2 b}{f^{\prime \prime}\left(w_{+}\right)}} \sqrt{1-u b}+\cdots
$$

Combining (4.5) and (4.6), we have 


$$
R\left(\frac{1}{u}, x\right)=B_{0}+B_{1} \sqrt{1-u b}+\cdots \quad \text { for } u \text { near } 1 / b
$$

where $B_{0}=w_{+}^{-1} A_{0}$ and $B_{1}=\left(-w_{+}^{-2} A_{0}+w_{+}^{-1} A_{1}\right) \sqrt{2 b / f^{\prime \prime}\left(w_{+}\right)}(<0)$. If $x=e$, then $R(1 / u, e)=1 / w$ is given by an expansion (4.7) with $B_{0}=w_{+}^{-1}$ and $B_{1}=-w_{+}^{-2} \sqrt{2 b / f^{\prime \prime}\left(w_{+}\right)}$. In case (i) of Theorem 2 , Lemma 5 shows that $1 / b$ is the only singularity of $R(1 / u, x)$ for $|u|=1 / b$, and so Darboux' Theorem [3, p. 498], [21, Theorem 8.8.4] shows that (4.1) holds with $\phi(x)=B_{1} / \Gamma(-1 / 2)$ $=-B_{1} / 2 \sqrt{\pi}$.

In case (ii) of Theorem $2, R(1 / u, x)$ is clearly an odd or even function depending on whether $|x|$ is even or odd. So if $|x|$ is odd, we have $R(1 / u, x)=B_{0}+B_{1} \sqrt{1+u b}+\cdots$ for $u$ near $-1 / b$, and if $|x|$ is even, we have $R(1 / u, x)=-B_{0}-B_{1} \sqrt{1+u b}+\cdots$ for $u$ near $-1 / b$. Thus Darboux's Theorem implies that (4.1) holds with $\phi(x)=-B_{1} b / \sqrt{\pi}$ if $n \equiv|x| \bmod 2$.

Finally, in case (iii) of Theorem 2, we have

$$
r_{j}(w)=\left(-w+\sqrt{\left.w^{2}+4 \beta_{j}^{2}\right)} / 2 \beta_{j}\right.
$$

and we can solve equation (4.3) explicitly (see $\S 4.3$ ) to obtain $w=$ $z^{-1}\left[\left(z^{2}-\left(\beta_{1}-\beta_{2}\right)^{2}\right)\left(z^{2}-1\right)\right]^{1 / 2}$.

We find that $r_{j}(w)=-w / 2 \beta_{j}+\phi_{j}(z)$ and $w=g(z)(z-1)^{1 / 2}$, where $\phi_{j}(z)$ and $g(z)$ are analytic in a neighbourhood of $z=1$, and $\phi_{j}(1)=1$ and $g(1)=\sqrt{8 \beta_{1} \beta_{2}}$. It follows that $R(1 / u, x)$ has an expansion

$$
R\left(\frac{1}{u}, x\right)=\frac{1}{\sqrt{8 \beta_{1} \beta_{2}}}(1-u)^{-1 / 2}+B_{0}+B_{1}(1-u)^{1 / 2}+\cdots
$$

for $u$ near 1 . The rest of the proof of case (iii) follows as for case (ii).

Remark. Another natural choice for the probability on $G$ is $p^{\prime}=$ $\sum \alpha_{j} p_{j}^{\prime}$, where $p_{j}^{\prime}(x)=1 / r_{j}$ for all $x \in G_{j}$. Since

$$
p^{\prime}=\left(\sum_{j} \alpha_{j} r_{j}^{-1}\right) \delta_{e}+\sum_{j} \alpha_{j}\left(r_{j}-1\right) r_{j}^{-1} p_{j},
$$

it is easy to derive from Theorem 2 the corresponding result for $p^{\prime}$.

Let us conclude this section by pointing out that in two cases we can solve the equation (4.3) explicitly, and thus find the resolvent and the Plancherel measure.

§ 4.2. When $|J|=n<\infty$ and $\alpha_{j}=1 / n$ and $r_{j}=r$ for all $j$, equation (4.3) becomes 


$$
z=\left(1-\frac{n}{2}\right) w+\frac{r-2}{2(r-1)}+\frac{n}{2} \sqrt{w^{2}-\frac{2(r-2)}{n(r-1)} w+\frac{r^{2}}{n^{2}(r-1)^{2}}}
$$

We can solve this for $S(z)=1 / w$ and obtain

$$
S(z)=\frac{-(n-2) z-\frac{r-2}{r-1}+n \sqrt{\left(z-\gamma_{+}\right)\left(z-\gamma_{-}\right)}}{2(z-1)\left(z+\frac{1}{r-1}\right)}
$$

where

$$
\gamma_{ \pm}=\frac{1}{n}\left[\frac{r-2}{r-1} \pm 2 \sqrt{\frac{n-1}{r-1}}\right]
$$

The square root must behave like $z$ as $z \rightarrow \infty$, and so is positive on $\left(\gamma_{+}, \infty\right)$ and negative on $\left(-\infty, \gamma_{-}\right)$. It follows that $S$ has a removable singularity at 1 , while $-1 /(r-1)$ is removable if and only if $n \geqq r$. If $n<r,-1 /(r-1)\left(<r_{-}\right)$is a pole with residue $1-n / r$. The Stieltjes inversion formula shows that the Plancherel measure is supported on $\left[\gamma_{-}, \gamma_{+}\right]$if $n \geqq r$ and on $\left[\gamma_{-}, \gamma_{+}\right] \cup\{-1 /(r-1)\}$ if $n<r$. On $\left[\gamma_{-}, \gamma_{+}\right], \mu$ is absolutely continuous with respect to Lebesgue measure, with density

$$
\frac{n \sqrt{\left(\gamma_{+}-x\right)\left(x-\gamma_{-}\right)}}{2 \pi(1-x)(x+1 /(r-1))}
$$

(cf. [7], [8], [14]).

$\S$ 4.3. When $|J|=2$, write $\alpha=\beta_{1}, \beta=\beta_{2}, r=r_{1}, s=r_{2}, x_{0}=(\alpha(r-2)$ $+\beta(s-2)) / 2$ and $t=z-x_{0}$. Then (4.3) becomes

$$
2 t=\sqrt{w^{2}-2 \alpha(r-2) w+\alpha^{2} r^{2}}+\sqrt{w^{2}-2 \beta(s-2) w+\beta^{2} s^{2}},
$$

and solving for $S(z)=1 / w$, we obtain

$$
S(z)=\frac{-x_{0} t^{2}+(\alpha(r-2)-\beta(s-2))\left(\alpha^{2} r^{2}-\beta^{2} s^{2}\right) / 8+t Q(t)}{\left(t^{2}-\left(\frac{\alpha r-\beta s}{2}\right)^{2}\right)\left(t^{2}-\left(\frac{\alpha r+\beta s}{2}\right)^{2}\right)},
$$

where $Q(t)$ is the square root of

$$
\begin{aligned}
& t^{4}-\frac{1}{2}\left((\alpha r-\beta s)^{2}+4 \alpha \beta(r+s-2)\right) t^{2} \\
& +(\alpha r-\beta s)^{4} / 16+\alpha \beta\left[(r+s-2)(\alpha r-\beta s)^{2}+2 \alpha \beta(r-s)^{2}\right] / 2 \\
& \quad=\left(t^{2}-\rho_{+}\right)\left(t^{2}-\rho_{-}\right)
\end{aligned}
$$

where 


$$
\rho_{ \pm}=\left(\frac{\alpha r-\beta s}{2}\right)^{2}+\alpha \beta(r+s-2) \pm 2 \alpha \beta \sqrt{(r-1)(s-1)} .
$$

Notice that $0 \leqq \rho_{-}<\rho_{+}$. Let $\gamma$ and $\delta$ be the positive square roots of $\rho_{-}$ and $\rho_{+}$respectively. Now $Q(t)$ must be positive if $|t|>\delta$ and negative if $|t|<\gamma$. It follows that $S$ has a removable singularity at $t=(\alpha r+\beta s) / 2$, a pole $t=-(\alpha r+\beta s) / 2$ with residue $1-1 / r-1 / s$, and a pole with residue $|1 / r-1 / s|$ at either $\mid(\alpha r-\beta s) / 2$, if $(r-s)(\alpha r-\beta s) \leqq 0$, or at $-|(\alpha r-\beta s) / 2|$, if $(r-s)(\alpha r-\beta s) \geqq 0$. By the Stieltjes Inversion formula, the Plancherel measure $\mu$ is supported by $\left[x_{0}-\delta, x_{0}-\gamma\right] \cup\left[x_{0}+\gamma, x_{0}+\delta\right] \cup\left\{x_{1}, x_{2}\right\}$, where $x_{1}=x_{0}-(\alpha r+\beta s) / 2=-(\alpha+\beta)$ and $x_{2}=x_{0}+(\alpha r-\beta s) / 2$ or $x_{0}-(\alpha r-\beta s) / 2$. For $\gamma \leqq\left|x-x_{0}\right| \leqq \delta, \mu$ is absolutely continuous with respect to Lebesgue measure, with density

$$
\frac{\left|x-x_{0}\right| \sqrt{\left(\delta^{2}-\left(x-x_{0}\right)^{2}\right)\left(\left(x-x_{0}\right)^{2}-\gamma^{2}\right)}}{\pi\left(\left(\frac{\alpha r+\beta s}{2}\right)^{2}-\left(x-x_{0}\right)^{2}\right)\left(\left(x-x_{0}\right)^{2}-\left(\frac{\alpha r-\beta s}{2}\right)^{2}\right)} \cdot
$$

Finally, $\mu\left(\left\{x_{1}\right\}\right)=1-1 / r-1 / s$ and $\mu\left\{x_{2}\right\}=|1 / r-1 / s|$ (cf. [5]).

\section{§5. The resolvent for quotient groups and amalgams}

$\S$ 5.1. Let $G$ be the free product with amalgamation $*_{H} G_{j}$ of a finite or countable family of discrete groups $G_{j}$ having a common finite normal subgroup $H$. There is a natural isomorphism between $G / H$ and the free product of the groups $G_{j} / H$. We shall now show that the study of the random walk associated with an $H$-invariant probability on $G$ can thus be reduced to the study of a random walk on this free product. To this end, it is convenient to generalize the problem and consider any discrete group $G$ having a finite normal subgroup $H$. We also consider a measure on $G$ which is a convex combination of an $H$-invariant probability on $G$ and an arbitrary probability on the finite subgroup $H$.

Thus let $G$ be a discrete group with finite normal subgroup $H$ of order $d$. We write $\bar{x}$ for $x H \in G / H$. Let $\tilde{p}_{1}$ be a probability measure on $G / H$, with resolvent $\tilde{R}_{1}(z)$. Define a probability $p_{1}$ on $G$ by $p_{1}(x)=(1 / d) \tilde{p}_{1}(\bar{x})$. Let $p_{2}$ be a probability measure on $H$ with resolvent $R_{2}(z)$. We define $p_{2}(x)=0$ for $x \in G \backslash H$. Let $\alpha, \beta \geqq 0$ with $\alpha+\beta=1$, and let $p(x)=\alpha p_{1}(x)$ $+\beta p_{2}(x)$. Denote by $\tilde{X}_{1}, X_{2}$ and $X$ the spectra of $\tilde{p}_{1}, p_{2}$ and $p$ respectively.

THEOREM 3. Let $R(\boldsymbol{z})$ be the resolvent of the probability measure $p$ described above. If $\alpha, \beta>0$ and if $z \notin\left(\beta+\alpha \tilde{X}_{1}\right) \cup \beta X_{2}$ then 


$$
R(z, x)=\left\{\begin{array}{l}
\alpha^{-1} d^{-1} \tilde{R}_{1}((z-\beta) / \alpha, \bar{x}) \quad \text { if } x \notin H \\
\alpha^{-1} d^{-1}\left[\tilde{S}_{1}((z-\beta) / \alpha)-\alpha /(z-\beta)+\alpha d \beta^{-1} R_{2}\left(z \beta^{-1}, x\right)\right] \\
\text { if } x \in H .
\end{array}\right.
$$

If $\alpha=1$ and $\beta=0$, and if $z \notin \tilde{X}_{1} \cup\{0\}$, then

$$
R(z, x)= \begin{cases}d^{-1} \tilde{R}_{1}(z, \bar{x}) & \text { if } x \notin H \\ d^{-1} \tilde{S}_{1}(z)-d^{-1} z^{-1}+z^{-1} \delta_{e}(x) & \text { if } x \in H .\end{cases}
$$

Proof. Write $\lambda=(z-\beta) / \alpha$. Let $f(x)$ denote the function defined by the right hand side of (5.1). Notice that $f(h x)=f(x)$ if $h \in H$ and $x \in G \backslash H$ and that $\sum_{h \in H} f(h)=\tilde{S}_{1}(\lambda) / \alpha$. Let $V$ be a set of coset representations of $H$ in $G$. Then

$$
\begin{aligned}
\left(p_{1} * f\right)(x) & =\sum_{v \in V} \sum_{h \in H} p_{1}(v h) f\left(h^{-1} v^{-1} x\right) \\
& =\frac{1}{\alpha \bar{d}} \sum_{v \in V, \bar{v} \neq \bar{x}} \tilde{p}_{1}(\bar{v}) \tilde{R}_{1}\left(\lambda, \bar{v}^{-1} \bar{x}\right)+\tilde{p}_{1}(\bar{x}) \tilde{S}_{1}(\lambda) / \alpha d \\
& =\frac{1}{\alpha d}\left(\tilde{p}_{1} * \tilde{R}_{1}(\lambda)\right)(\bar{x})
\end{aligned}
$$

Also,

$$
\begin{aligned}
(p * f)(x) & =\sum_{h \in H} p_{2}(h) f\left(h^{-1} x\right) \\
& = \begin{cases}f(x)=\frac{1}{\alpha d} \tilde{R}_{1}(\lambda, \bar{x}) & \text { if } x \in G \backslash H \\
\left(p_{2} * f\right)(x) & \text { if } x \in H .\end{cases}
\end{aligned}
$$

Thus

$$
(p * f)(x)=\frac{1}{d}\left(\tilde{p}_{1} * \tilde{R}_{1}(\lambda)\right)(\bar{x})+\frac{\beta}{\alpha d} \tilde{R}_{1}(\lambda, \bar{x})=z f(x) \quad \text { if } x \notin H .
$$

If $x \in H$, then

$$
\begin{aligned}
\left(p_{k}^{*} * f\right)(x) & =\frac{1}{d}\left(\tilde{p}_{1} * \tilde{R}_{1}(\lambda)\right)(\bar{e})+\beta\left(p_{2} * f\right)(x) \\
& =d^{-1}\left(\lambda \tilde{S}_{1}(\lambda)-1\right)+\beta\left[\frac{1}{\alpha d}\left(\tilde{S}_{1}(\lambda)-\frac{1}{\lambda}\right)+\frac{1}{\beta}\left(p_{2} * R_{2}\left(\frac{z}{\beta}\right)\right)(x)\right] \\
& =\frac{z}{\alpha d}\left(\tilde{S}_{1}(\lambda)-\frac{1}{\lambda}\right)+\left(p_{2} * R_{2}\left(\frac{z}{\beta}\right)\right)(x) \\
& =z f(x)-\delta_{e}(x) .
\end{aligned}
$$

Now notice that $f$ is the sum of two functions, $f=\phi+\psi$, say, where $\phi$ has support in $H$ and $\psi$ is $H$-invariant and corresponds to a bounded 
convolution operator on $\ell^{2}(G / H)$. Standard arguments show that $f$ defines a bounded convolution operator on $\ell^{2}(G)$. This proves the first part of the theorem. The second part is proved in exactly the same way or by taking limits in (5.1) and $\beta \rightarrow 0$.

Now let $G_{j}$ be a family of discrete groups containing a common normal finite subgroup $H$. Let $G$ be the amalgamated product $*_{H} G_{j}$ and $F$ the free product $* G_{j} / H$. Let $q_{j}$ be an $H$-invariant probability measure on $G_{j}$ for each $j$, and $p_{1}=\Sigma \alpha_{j} q_{j}$ where $\alpha_{j}>0$ and $\Sigma \alpha_{j}=1$. Write $\tilde{p}_{1}$ and $\tilde{q}_{j}$ for the corresponding probabilities on $F$ and $G_{j} / H$ respectively. Moreover, let $p_{2}$ be a probability measure on $H$ and form $p=\alpha p_{1}+\beta p_{2}$.

CoRollaRy. The resolvent of the probability measure $p$ on $G=*_{H} G_{j}$ is given by Theorem 3 , where the resolvent $\tilde{R}_{1}$ of $\tilde{p}_{1}$ is expressed in terms of the resolvents of the $\tilde{q}_{j}$ using Theorem 1 .

Before giving an example of the situation described in the corollary, let us make some remarks about the spectrum $X$ of $p$.

By Theorem 3, we have $X \subseteq\left(\beta+\alpha \tilde{X}_{1}\right) \cup \beta X_{2}$. If the probabilities $\tilde{p}_{1}$ and $p_{2}$ of Theorem 3 are symmetric, we can be more specific. Indeed, let $\tilde{\mu}_{1}, \mu_{2}$ and $\mu$ be the Plancherel measures of $\tilde{p}_{1}, p_{2}$ and $p$ respectively. For the sake of clarity we state explicitly the following elementary lemma which describes $\mu_{2}$.

Lemma. Let $\lambda_{1}=1, \lambda_{2}, \cdots, \lambda_{r}$ be the distinct eigenvalues of the convolution operator $f \mapsto p_{2} * f$ on $\ell^{2}(H)$, with corresponding multiplicities $n_{1}(\geqq 1), n_{2}, \cdots, n_{r}$. Then $\mu_{2}=\sum_{j=1}^{r}\left(n_{j} / d\right) \delta_{\lambda_{j}}$. Furthermore, $n_{1}=1$ if and only if the support of $p_{2}$ generates $H$.

Proof. Let $q_{j}$ be the function in the algebra $A^{*}$ generated by $p_{2}$ such that $\hat{q}_{j}\left(\lambda_{k}\right)=\delta_{j k}$. Then clearly $R_{2}(z)=\sum_{1}^{r}\left(z-\lambda_{j}\right)^{-1} q_{\jmath}$, and so $\mu_{2}=$ $\sum_{1}^{r} q_{j}(e) \delta_{\lambda_{j}}$. On the other hand, $f \mapsto q_{j} * f$ is the orthogonal projection $\ell^{2}(H)$ onto $V_{j}=\left\{f: p_{2} * f=\lambda_{j} f\right\}$, and thus $n_{j}=$ Trace $\left(q_{j}\right)=\sum_{x \in H}\left\langle q_{j} * \delta_{x}, \delta_{x}\right\rangle=$ $d q_{j}(e)$. If $K$ is the subgroup of $H$ generated by the support of $p_{2}$, then the characteristic function of any right coset $K x$ of $K$ in $H$ is in $V_{1}$. Thus $n_{1} \geqq|H| /|K|$. To see that equality holds see, e.g., Proposition I. 6.2 of [18].

Corollary 1. If $\tilde{p}_{1}$ and $p_{2}$ in Theorem 3 are symmetric and if $\alpha, \beta>0$, then the Plancherel measure of $p$ is given by

$$
\mu=\frac{1}{d} \nu+\frac{n_{1}-1}{d} \delta_{\beta}+\sum_{2}^{r} \frac{n_{j}}{d} \delta_{\beta \lambda_{j}},
$$


where $\nu(A)=\tilde{\mu}_{1}((A-\beta) / \alpha)$ for any Borel set $A \subseteq \boldsymbol{R}$. If, however, $\alpha=1$ and $\beta=0$, then

$$
\mu=\frac{1}{d} \tilde{\mu}_{1}+\left(1-\frac{1}{d}\right) \delta_{0}
$$

Proof. If we take $x=e$ in (5.1) and use the lemma, we see that

$$
\int_{R} \frac{d \mu(t)}{z-t}=\frac{1}{d} \int_{R} \frac{d \tilde{\mu}_{1}(t)}{z-(\alpha t+\beta)}+\frac{\left(n_{1}-1\right) / d}{z-\beta}+\sum_{2}^{r} \frac{n_{j} / d}{z-\beta \lambda_{j}}
$$

when $\alpha, \beta>0$. Thus (5.3) follows from the Stieltjes Inversion Formula. For the same reasons, (5.4) holds.

Corollary 2. With notation as above, if $\alpha, \beta>0$ then the spectrum $X$ of $p$ is $\left(\alpha \tilde{X}_{1}+\beta\right) \cup \beta X_{2}$, with the possible exception of the point $\beta$ (if $n_{1}=1$ and $\left.0 \notin \tilde{X}_{1}\right)$. The spectral radius $r$ of $p$ is $\alpha \tilde{r}_{1}+\beta$ if $\tilde{r}_{1}$ is that of $\tilde{p}_{1}$.

Proof. This is immediate from formulas (5.3) and (5.4).

\section{§5.2. Application to local limit theorems}

Let us briefly point out how Theorem 3 may be used to obtain a local limit theorem for $p$ if sufficient is known about the resolvent of $\tilde{p}_{1}$.

In the notation of Theorem 3 , if $\alpha=1$ and $\beta=0$, we see from (5.2), or directly, that $p^{* n}(x)=d^{-1} \tilde{p}_{1}^{* n}(\bar{x})$ for all $x \in G$ and $n \geqq 1$. The asymptotic behaviour of $p^{* n}(x)$ as $n \rightarrow \infty$ is therefore known once we know that of $\tilde{p}_{1}^{* n}(\bar{x})$.

When $\alpha, \beta>0$, the relationship between $p^{* n}(x)$ and $\tilde{p}_{1}^{* n}(\bar{x})$ and $p_{1}^{* n}(x)$ is more complicated. Suppose, for simplicity, that $\tilde{p}_{1}$ is symmetric. If $\tilde{r}_{1}$ denotes the spectral radius of $\tilde{p}_{1}$, then it is clear from (5.1) that $R(z, x)$ is analytic for $|z|>r=\beta+\alpha \tilde{r}_{1}$. Furthermore, $z=r$ is the only singularity of $R(z, x)$ on the circle $|z|=r$, because $\left|\beta-\alpha \tilde{r}_{1}\right|<r$ and $\beta<r$. Suppose now that, as was the case in Theorem $2, \tilde{R}_{1}(1 / z, \bar{x})$ has a Puiseux expansion $\tilde{R}_{1}(1 / z, \bar{x})=B_{0}+B_{1} \sqrt{1-z \tilde{r}_{1}}+\cdots$ for $z$ near $1 / \tilde{r}_{1}$ where $B_{1}=B_{1}(\bar{x})$ $<0$. Then by (5.1), $R(1 / z, x)$ has a Puiseux expansion $R(1 / z, x)=C_{0}+$ $C_{1} \sqrt{1-z r}+\cdots$ for $z$ near $1 / r$, where $C_{1}=\alpha^{-1} d^{-1} B_{1}(1-\beta / r)^{-1 / 2}$. By Darboux's Theorem again, we have

$$
p^{* n}(x)=\frac{-C_{1}}{2 \sqrt{\pi}} n^{-3 / 2} r^{n}\left[1+O\left(\frac{1}{n}\right)\right] \quad \text { as } n \rightarrow \infty .
$$




\section{§ 5.3. Application to orthogonal polynomials}

Let us consider the sequence $\left\{p_{n}\right\}$ of orthogonal polynomials associated with the Plancherel measure $\mu$ of a symmetric probability $p$ on a discrete group $G$. For $G$ and $p$ as in Section 4.2 , the polynomials $\left\{p_{n}\right\}$ have been studied in [7] and [14], and they are shown to be combinations of Chebyshev polynomials. The polynomials corresponding to the $G$ and $p$ of Section 4.3 (with $\alpha=\beta=1$ ) are more complicated and were described in $[5]$.

When $G$ and $p$ are as in Theorem 3, the Plancherel measure $\mu$ is obtained from that of $\tilde{p}_{1}$ by Corollary 1 above, and so the orthogonal polynomials associated with $\mu$ may be calculated by successive applications of the following general proposition.

Proposition. Let $\left\{q_{n}\right\}$ be the monic orthogonal polynomials relative to a measure $\nu$ on $R$. Form the measure $\mu=a \nu+b \delta_{c}$, where $a, b>0$, $a+b=1$ and where $\delta_{c}$ is the unit mass at $c \in \boldsymbol{R}$. Then the monic orthogonal polynomials $\left\{p_{n}\right\}$ relative to $\mu$ are given by

$$
\begin{aligned}
& p_{n}(x)=\sigma_{n}\left[\sum_{k=0}^{n-1} \frac{q_{k}(c)}{\left\|q_{k}\right\|^{2}} q_{k}(x)\right]+q_{n}(x) \quad \text { in } n \geqq 1 \\
& =\frac{\sigma_{n}}{\left\|q_{n-1}\right\|^{2}}\left[\frac{q_{n}(x) q_{n-1}(c)-q_{n-1}(x) q_{n}(c)}{x-c}\right]+q_{n}(x) \quad \text { if also } x \neq c,
\end{aligned}
$$

where $\left\|q_{k}\right\|^{2}=\int_{R} q_{k}(x)^{2} d \nu(x)$ and

$$
\sigma_{n}=-b q_{n}(c)\left[a+b \sum_{k=0}^{n-1} \frac{q_{k}(c)^{2}}{\left\|q_{k}\right\|^{2}}\right]^{-1} .
$$

Proof. Define $p_{n}$ for $n \geqq 1$ by the first equation in (5.5), where $\sigma_{n}$ is given by (5.6). For $n \geqq 1$, we have

$$
\begin{aligned}
\int_{R} p_{n}(x) d \mu & =a \int_{R} p_{n}(x) d \nu+b p_{n}(c) \\
& =a \sigma_{n}+b \sigma_{n}\left[\sum_{0}^{n-1} q_{k}(c)^{2}\left\|q_{k}\right\|^{-2}\right]+b q_{n}(c) \\
& =0 .
\end{aligned}
$$

Furthermore, if $n>m \geqq 1$, then

$$
\begin{aligned}
\int_{R} p_{n}(x) p_{m}(x) d \mu & =a \int_{R} p_{n}(x) p_{m}(x) d \nu+b p_{n}(c) p_{m}(c) \\
& =a \sigma_{n} \sigma_{m}\left[\sum_{0}^{m-1} \frac{q_{k}(c)^{2}}{\left\|q_{k}\right\|^{2}}\right]+a \sigma_{n} q_{m}(c)+b p_{n}(c) p_{m}(c)
\end{aligned}
$$




$$
\begin{aligned}
& =a \sigma_{n} p_{m}(c)+b p_{n}(c) p_{m}(c) \\
& =0
\end{aligned}
$$

because $a \sigma_{n}+b p_{n}(c)=0$. Thus $\left\{p_{n}\right\}$ are the monic orthogonal polynomials for $\mu$. The second equation in (5.5) follows from the first by the ChristoffelDarboux formula [21, Theorem 3.2.2].

Example. Let $G$ be the free product of $n$ copies of the cyclic group $Z_{r d}$ with the common subgroup $Z_{d}$ amalgamated. Define $p$ by $p(x)=$ $1 / d n(r-1)$ if $x$ is an element of one of the copies of $Z_{r d}$ but $x \notin Z_{d}$, and set $p(x)=0$ for all other $x \in G$. Then $p(x)=(1 / d) \tilde{p}_{1}(\bar{x})$, where $\tilde{p}_{1}$ is the probability on $G / Z_{d}$ (= the free product $n$ copies of $Z_{r}$ ) discussed in Section 4.2. The Plancherel measure is given by (5.4) and the orthogonal polynomials $\left\{q_{n}\right\}$ for $\tilde{\mu}_{1}$ may be expressed simply as a combination of Chebyshev polynomials of the second kind (see the lemma in [14]). Thus by the above proposition with $\nu=\tilde{\mu}_{1}, c=0, a=1 / d$ and $b=1-1 / d$, the orthogonal polynomials $\left\{p_{n}\right\}$ for the Plancherel measure $\mu$ of $p$ are explicitly expressible in terms of these Chebyshev polynomials.

ExAmple. $S L_{2}(Z) \cong Z_{4} *_{Z_{2}} Z_{6}[19$, p. 11]. The orthogonal polynomials for a natural probability on $S L_{2}(Z) / Z_{2} \cong Z_{2} * Z_{3}$ are described in [5]. Thus the above proposition describes the orthogonal polynomials for the corresponding $Z_{2}$-invariant probability on $S L_{2}(Z)$.

\section{Note.}

During the final preparation of this manuscript, the authors were informed by $\mathrm{W}$. Woess that he had also recently proved a formula for the resolvent for probabilities on the free product of discrete groups. His methods, which involve the study of several auxiliary functions, and his applications are different however.

\section{References}

[1] K. Aomoto, Spectral theory on a free group and algebraic curves, J. Fac. Sci. Univ. Tokyo Sec. 1A, 31 (1984), 297-317.

[2] K. Aomoto and Y. Kato, Green functions and spectrums in a free product of cyclic groups, preprint.

[3] E. A. Bender, Asymptotic methods in enumeration, SIAM Review, 16 (1974), 485-515.

[ 4 ] P. Cartier, Fonctions harmoniques sur un arbre, Symposia Math., 9 (1972), 203270.

[ 5 ] D. I. Cartwright and P. M. Soardi, Harmonic analysis on the free product of two cyclic groups, J. Funct. Anal., 65 (1986), 147-171. 
[6] T. S. Chihara, An introduction to orthogonal polynomials, Gordon and Breach, New York-London-Paris, 1978.

[ 7 ] J. M. Cohen and A. R. Trenholme, Orthogonal polynomials with a constant recursion formula and an application to harmonic analysis, J. Funct. Anal., 59 (1984), 175-184.

[8] J. Faraut and M. A. Picardello, The Plancherel measure for symmetric graphs, Ann. Mat. Pura Appl., 138 (1984), 151-155.

[9] A. Figà-Talamanca and M. A. Picardello, Harmonic analysis on free groups, Lecture Notes in Pure and Applied Mathematics, 87, Marcel Dekker, New York, 1983.

[10] F. Gerl, Ein Gleichverteilungssatz auf $F_{2}$, Probability Measures on Groups, Lecture Notes in Math., 706, Springer-Verlag, Berlin-Heidelberg-New York, 1979, pp. $126-130$.

[11] - A local central limit theorem on some groups, The First Pannonian Symposium on Mathematical Statistics, Lecture Notes in Statistics 8, Springer-Verlag, Berlin-Heidelberg-New York, 1981, pp. 73-82.

[12] - and W. Woess, Local limits and harmonic functions for non-isotropic random walks on free groups, Probab. Th. Rel. Fields, 71 (1986), 341-355.

[13] H. Kesten, Symmetric walks on groups, Trans. Amer. Math. Soc., 92 (1959), $336-354$.

[14] G. Kuhn and P. M. Soardi, The Plancherel measure for polygonal graphs, Ann. Mat. Pura Appl., 134 (1983), 393-401.

[15] M. A. Picardello, Spherical functions and local limit theorems on free groups, Ann. Mat. Pura Appl., 133 (1983), 177-191.

[16] M. A. Picardello and W. Woess, Random walks on amalgams, Monatsh. Math., 100 (1985), 21-33.

[17] S. Sawyer, Isotropic random walks in a tree, Z. Wahrsch. Verw. Gebiete, 42 (1978), $279-292$.

[18] H. H. Schaefer, Banach lattices and positive operators, Grundlehren der Math. Wissen. 215, Springer-Verlag, Berlin-Heidelberg-New York, 1974.

[19] J. P. Serre, Trees, Springer-Verlag, Berlin-Heidelberg-New York, 1980.

[20] P. M. Soardi, The resolvent for simple random walks on the free product of two discrete groups, preprint.

[21] G. Szegö, Orthogonal Polynomials, Amer. Math. Soc. Colloq. Publications, Vol. XXIII, fourth edition, Amer. Math. Soc., Providence Rhode Island, 1975.

[22] W. Woess, A random walk on the free product of finite groups, in Springer Lecture Notes in Mathematics 1064, (1984), 467-470.

Donald I. Cartwright

Department of Pure Mathematics

The University of Sydney

Sydney N.S.W. 2006

Australia

P. M. Soardi

Dipartimento di Matematica

Università di Milano

Via C. Saldini 50

Milano, 20133

Italy 